\title{
Universidades, diálogo de saberes y sustentabilidad. La UNICh ante el desarrollo local, alcances y retos
}

DOI: http://dx.doi.org/10.25087/resur3a3

\author{
Liberio Victorino Ramírez ${ }^{1}$ \\ Rosey Obet Ruiz González 2 \\ María del Rosario González Hernández ${ }^{3_{3}}$
}

Recibido, 23 de junio de 2017

Aprobado, 24 de julio de 2017

\section{Resumen}

La propuesta académica del modelo educativo intercultural demanda un compromiso con la sociedad ya que parten del reconocimiento y valoración de los saberes tradicionales y modernos. En ese sentido, los esquemas tradicionales en que el profesor es el centro del proceso educativo resultan obsoletos para situarse ahora en los estudiantes y en la diversidad de saberes en la que ellos se desenvuelven. La universidad intercultural ha desarrollado una propuesta que incluye un diálogo de los saberes adquiridos en el aula (saberes de los cursos), los saberes de los/las estudiantes (saberes propios) y los saberes locales (saberes de las comunidades).

El objetivo de este artículo consiste en comparar su capacidad de diálogo de saberes, valorar sus alcances respecto al modelo curricular, así como ubicar sus principales retos a superar en el futuro inmediato. La metodología empleada tiene como trayecto dos momentos muy importantes: uno, que se ubica en el plano del diseño curricular que nos habla de la concepción del modelo planteado; dos, el que tiene que tomar en cuenta el nivel de resultados que obtuvieron a sus 10

${ }^{1}$ Profesor-Investigador, Universidad Autónoma Chapingo. Miembro del Sistema Nacional de Investigadores.

CE:victorinoramrezliberio@yahoo.com.mx

${ }^{2}$ Doctor en Ciencias en Educación Agrícola. CE: superior. mcobet.10@gmail.com

${ }^{33}$ Doctora en Educación Agrícola Superior, Universidad Autónoma Chapingo. CE: charo_137@yahoo.com.mx

Rev. educ. super. sur glob-RESUR N³ $\quad$ En.-jul 2017 e006 ISSN 2393-6789 
años de fundación del modelo curricular en el contexto de desigualdad social en el estado de Chiapas.

Palabras clave: interculturalidad, diálogo de saberes, desarrollo sustentable

\begin{abstract}
The academic proposal of the intercultural educational model demands a commitment to society as it starts from the recognition and appreciation of traditional and modern knowledge. In that sense, the traditional schemes in which the teacher is the center of the educational process are now obsolete leaving it place to students and the diversity of knowledge. The intercultural university has developed a proposal that includes a dialogue of the knowledge acquired in the classroom (knowledge of the courses), the knowledge of the students (own knowledge) and local knowledge (knowledge of the communities).

The objectives of this article are to compare its capacity for dialogue of knowledge, to evaluate its scope regarding to the curricular model, and to identify its main challenges to overcome in the immediate future. The methodology used has two important moments: one, located at the level of curricular design, which tells us about the conception of the model proposed; two, the level of results obtained after 10 years of implementation of the curricular model in the context of social inequality in the state of Chiapas.
\end{abstract}

Key words: interculturality, dialogue of knowledge, sustainable development

\title{
Introducción
}

La interculturalidad es el elemento distintivo de nuestro modelo de educación superior. La interculturalidad designa la dinámica de las relaciones sociales en el contexto de la diversidad cultural y lo intercultural, tiene que ver con las prácticas culturales y modos de vida concretos de las personas que se ponen en interacción con otras mediante el diálogo y la interacción entre culturas. El mecanismo que pone en acción la interculturalidad, que es la forma más desarrollada

Rev. educ. super. sur glob-RESUR N³ En.-jul 2017 e006 ISSN 2393-6789 
del diálogo de las culturas y las civilizaciones, es el "diálogo intercultural” (Barabas, 2006). El diálogo intercultural implica necesariamente un diálogo de saberes como producto de la interacción social. Es por ello la relación y pertinencia con el desarrollo sustentable ya que al buscar un equilibrio en el desarrollo se extiende necesariamente hacia los saberes y las relaciones sociales. Desde luego, este diálogo implica una relación de lo social, lo económico y lo cultural y por su diversidad de enfoques, lo convierten en un paradigma.

El paradigma del desarrollo así como su interpretación y aplicación a contextos específicos ha sido objeto de un largo y profundo debate en todo tipo de foros, conferencias, encuentros y propuestas de desarrollo. El concepto ha estado influenciado por factores biológicos, económicos, sociales, culturales e incluso religiosos. Es conocido que a lo largo de la historia se han acuñado diversos conceptos para definir al desarrollo en sus diversas modalidades. De este modo, surgieron términos como progreso y crecimiento, entre otros que basan sus principios en torno a una "oferta infinita de recursos naturales" cuya expresión máxima se alcanzó con la llamada Revolución Verde entre 1940 y 1960. Ante un panorama de alto uso de los recursos naturales, un acelerado crecimiento demográfico y la rápida colonización de zonas boscosas y selváticas, el discurso del desarrollo dominado en gran parte por la economía, no vislumbraba siquiera la posibilidad de los recursos naturales ante un panorama de creciente desarrollo industrial y tecnológico vinculado a la apertura de nuevos mercados.

En la década de 1980, el debate de la relación entre población y ambiente pronto fue el tema central de las conferencias y encuentros regionales e internacionales. El supuesto de la infinitud de los recursos naturales ya no tenía cabida como parte del discurso del desarrollo y había que tomar las previsiones necesarias para un debate serio. Es por ello que la noción de desarrollo vinculado a la sustentabilidad apareció por primera vez en 1987 durante la Conferencia sobre el Medio Ambiente y el Desarrollo, también conocida como Nuestro Futuro Común, misma que elaboró el célebre Informe Bruntland donde se habla de un desarrollo sustentable que se plantea como "un estilo de desarrollo que satisface las necesidades del presente sin comprometer

Rev. educ. super. sur glob-RESUR No3 En.-jul 2017 e006 ISSN 2393-6789 
las capacidades de las futuras generaciones" (ONU, 1987). En síntesis, el documento establece la búsqueda del equilibrio de los tres componentes o ejes fundamentales de la sustentabilidad: económica, ambiental y social.

Dicha propuesta ha favorecido un amplio debate que en el fondo implica el restablecimiento del equilibrio entre población y ambiente. Sin embargo, alcanzar un equilibrio socioeconómico y ambiental en el contexto del desarrollo capitalista se torna muy difícil por la desigualdad e inequidad que caracterizan al modelo de desarrollo desde su mismo origen y definición. El sistema capitalista actual está basado en un proceso de globalización económica y en el libre mercado sin restricciones donde el gran capital ha propiciado mayor desigualdad y pobreza, así como la creciente pérdida de la responsabilidad social del Estado.

Ante este panorama, la educación superior requiere retomar su función social y abrirse a una perspectiva que promueva el desarrollo integral del individuo y sus comunidades en armonía con el ambiente, pero sobre todo con una nueva visión ante los problemas y necesidades de la gente, así como el reconocimiento de los saberes locales. Es por ello que la Universidad Intercultural de Chiapas (UNICh), ha planteado sus ofertas educativas de manera que contribuya al desarrollo sustentable desde una perspectiva que favorezca el desarrollo local integral y a la revaloración de los saberes y competencias locales, así como un manejo territorial de los recursos naturales.

El objetivo de este trabajo es comparar las experiencias del modelo educativo intercultural, en torno a su capacidad de diálogo de saberes, valorar sus alcances del modelo curricular, así como ubicar sus principales retos a superar en el futuro inmediato.

\section{Metodología}

Dado el propósito planteado, adoptamos un método mixto de investigación utilizando la técnica cualitativa no experimental y la documental, la cual surge de las bases epistemológicas que determinan la construcción de las evidencias, los criterios a utilizar para formular los juicios de

Rev. educ. super. sur glob-RESUR N³ $\quad$ En.-jul 2017 e006 ISSN 2393-6789 
valor desde la perspectiva de la comprensión de las posibilidades de conocimiento de la realidad humana (Briones, 2002:113-125). Las fuentes consultadas fueron, en coherencia con la educación intercultural, generadas en el campo de la pedagogía, y la sociología, al tiempo que se recogieron reflexiones autores que publican en revistas indizadas dedicadas a la difusión de la investigación educativa, bases de datos y congresos nacionales que se llevaron a cabo en este sector educativo.

Esta parte metodológica incluye como trayectoria dos momentos muy importantes: uno, que se ubica en el plano del diseño curricular que nos habla la concepción del modelo planteado; dos, el que tiene que tomar en cuenta el nivel de resultados que se obtuvieron a sus 10 años de fundación del modelo curricular en el contexto de desigualdad social en el estado de Chiapas.

\section{Chiapas en el contexto del desarrollo nacional y educación superior}

El estado de Chiapas se localiza en la región sureste de México y limita con los estados de Oaxaca, Veracruz, Tabasco, Campeche, Quintana Roo y la República de Guatemala. Ocupa uno de los primeros lugares en biodiversidad natural y cultural; en lo social y económico, la historia de Chiapas da muestras de la explotación y desigualdad que ha prevalecido por siglos entre la población local, especialmente entre los sectores indígenas.

Chiapas es un estado reconocido a nivel mundial por su gran diversidad en recursos naturales, especies vegetales y animales, pero paradójicamente se ubica entre los estados con menor índice de desarrollo donde domina la pobreza y la marginación y donde la educación superior, entre otros aspectos, es poco accesible para la población joven del estado.

La problemática del desarrollo en Chiapas incluye aspectos económicos como la misma pobreza y marginación mencionada, los bajos rendimientos productivos, las políticas y programas poco acordes a las necesidades de los pueblos locales, así como la creciente migración a destinos nacionales e internacionales. En lo ambiental hay un gran deterioro de bosques y selvas, erosión de suelos, así como la pérdida de especies animales y vegetales. En lo social no deja de preocupar la falta de reconocimiento de la diversidad cultural y los derechos de los grupos autóctonos de

Rev. educ. super. sur glob-RESUR No3 En.-jul 2017 e006 ISSN 2393-6789 
Chiapas, además de la discriminación, desnutrición, mortalidad, los conflictos religiosos, agrarios e interétnicos, así como el limitado acceso a una educación superior de calidad.

En concreto, el estado de Chiapas enfrenta dos grandes problemáticas que requiere atender de forma inmediata y conjunta: una de ellas es el deterioro acelerado de los recursos naturales, especialmente en la última tres décadas, y otra es, la pérdida de su cultura, lengua, usos y costumbres y tradiciones de los grupos étnicos locales, impulsada en gran parte por los nuevos modelos económicos, que entre otros aspectos buscan la modernización de la sociedad.

\section{Región Altos de Chiapas}

La investigación se centró en la Universidad Intercultural, ubicada en el municipio de San Cristóbal de Las Casas de la región Altos de Chiapas. Esta región se caracteriza por tener una diversidad cultural por el devenir histórico de diversas culturas y una población en su mayoría indígena rural. Representa la región II de las regiones socioeconómicas del estado de Chiapas, siendo San Cristóbal de Las Casas la localidad sede. Según (Velasco, 2004), esta región, representa el $6 \%$ del total de la entidad $\left(3,770.8 \mathrm{~km}^{2}\right)$. Se sitúa a $16^{\circ} 15^{\prime \prime}$ y $17^{\circ} 07^{\prime \prime}$ de latitud norte y $91^{\circ} 32^{\prime \prime}$ y $93^{\circ} 05^{\prime \prime}$ de longitud oeste; colinda al sur con las regiones centro y fronteriza, al norte con las regiones norte y selva, al este con las regiones fronteriza y selva y al oeste con la región del centro y comprende 16 municipios: Chalchihuitán, Pantelhó, Chenalhó, Larrainzar, Chamula, Mitontic, Tenejapa, Oxchuc, Altamirano, Zinacantán, San Cristóbal de Las Casas, Huixtán, Chanal, Teopisca, Amatenango del Valle y Villa de las Rosas.

El total de la población es de 601,190 habitantes, representando el 12.53\% de la población estatal, convirtiéndola en la tercera región más poblada en el estado (INEGI, 2010). La distribución por sexo de la población se observa en el cuadro 1.

Cuadro 1. Distribución por sexo de la región Altos de Chiapas

Rev. educ. super. sur glob-RESUR $N^{o} 3 \quad$ En.-jul 2017 e006 $\quad$ ISSN 2393-6789 


\begin{tabular}{lll}
\hline Sexo & Absolutos & Porcentaje \\
\hline Masculino & 290,416 & 48.12 \\
Femenino & 310,774 & 51.88 \\
\hline
\end{tabular}

La región Altos de Chiapas, es la segunda más densamente poblada, con 203.13 habitantes por kilómetro cuadrado, con una tasa media anual de crecimiento de $1.77 \%$, una tasa global de fecundidad de $4.94 \%$, una tasa de mortalidad general de $4 \%$ y una tasa de mortalidad en menores de un año de 19.96\%. La tasa media anual de crecimiento de la región en el periodo 2005-2010 fue de $2.48 \%$, indicador que se encuentra por arriba del promedio estatal que es de $2.44 \%$. Los municipios que presentaron las tasas más bajas de crecimiento anual fueron Amatenango del Valle y Aldama con un promedio de 0.55 y $0.71 \%$ respectivamente. Y como se puede apreciar, la mayor parte de la población corresponde al sexo femenino. Las características de las viviendas de la región, se presenta en el cuadro 2 .

Cuadro 2. Características de las viviendas de la región Altos de Chiapas (2015)

\begin{tabular}{ll}
\hline Viviendas & Cantidad \\
\hline De tierra & 28,545 \\
Cemento o firme & 82,789 \\
Madera, mosaico u otro recubrimiento & 7,850 \\
Agua & 91,369 \\
Drenaje & 70,637 \\
Energía & 113,553 \\
\hline
\end{tabular}

Fuente: Programa Regional de Desarrollo. Región V Altos Tsotsil-Tseltal

Del total de la población de los Altos, 408,598 habitantes hablan alguna lengua indígena representando el $68 \%$ de la población regional (cuadro 3).

\begin{tabular}{lllll}
\hline Rev. educ. super. sur glob-RESUR $N^{\circ} 3$ & En.-jul 2017 e006 & ISSN 2393-6789
\end{tabular} 
Cuadro 3. Población indígena de los Altos de Chiapas (2015)

\section{Población indígena}

Región Altos de Chiapas

Población en hogares indígenas

Población de 3 años y más que habla lengua indígena

479,753

408,958

Población de 3 años y más bilingüe

200,557

Población de 3 años y más monolingüe
204,199

Fuente: Programa Regional de Desarrollo. Región V Altos Tsotsil-Tseltal

Con respecto a la educación escolar en la región, la situación económica ha sido un factor que incide en el rezago educativo, propiciando la inasistencia escolar. Las familias, al no contar con la suficiencia de recursos se encuentran imposibilitados para sufragar gastos para la compra de útiles escolares y uniformes, siendo en muchos casos necesario que niños y niñas se sumen al mercado laboral o bien a las labores del campo para contribuir con los ingresos de la familia.

El índice de analfabetismo en la región es de 26.8, muy por encima del resultado estatal que es de 17.8. En general, el grado promedio alcanzado en la Región es de 4.55, por género es 5.28 para los hombres y 3.88 para mujeres. Este dato se ve reflejado en una carencia por rezago educativo puesto que en la región, un $42 \%$ de la población sufre este flagelo. Un $90.8 \%$ de la población de 6-11 años asiste a la escuela; al igual que un 79.9\% de la población de 12-14 años. Para la población de 15-17 años, el resultado es de un 46.5\% y se reduce drásticamente a un $15.3 \%$ en la población de 18-24 años que asiste a la escuela. En la región existe mayor deserción en bachillerato, siendo los hombres quienes más desertan con 9.02\% y las mujeres con 1.99\%; a nivel primaria y secundaria la tendencia es cuanto a género es la misma aunque la tasa de deserción es de alrededor de 0.16 .

\section{El modelo educativo intercultural}

Rev. educ. super. sur glob-RESUR N³ $\quad$ En.-jul 2017 e006 ISSN 2393-6789 
La educación intercultural surgió como consecuencia de la confrontación de dos o más culturas dentro de una misma sociedad, fue un proceso que inició en los años sesenta cuando emergieron los movimientos de revitalización étnica. De acuerdo con Bruckmann y Dos Santos (2008:7), en los años setenta del siglo XX, en América Latina renace con fuerza el movimiento indígena, asumiendo el carácter de una postura ideológica como sujeto social importante (Victorino, 2016) para las luchas sociales contemporáneas y que en México alcanza una expresión clara en el neozapatismo (nos referimos al movimiento que se levantó en armas el 1 de enero, 1994, como repudio a la puesta en práctica el Tratado de Libre Comercia entre Canadá, Estados Unidos y México, entre otras inconformidades). Este movimiento latinoamericano pretende ser mundial, es decir, indígenas en diferentes regiones del orbe buscan afirmar sus luchas en una ideología opuesta al capitalismo, crisis que se da especialmente en los sistemas de educación formal. Durante los años setenta se dieron a conocer diversos análisis, reflexiones y propuestas; por un lado, penetraba más la raíz de la institución escolar y por otro, no se hacía una crítica puntual a determinadas escuelas, sino que era bastante global a la institución; pero lo claro era que la sola expansión del aparato escolar no servía como único recurso para las expectativas de formación y aprendizaje. Lo anterior hace necesario observar esta realidad negada en nuestra sociedad y nuestras universidades, donde el marco escolar no siempre es el más idóneo para atender todas las necesidades y demandas educativas que se presentan. Dentro de este marco, uno de los cambios más significativos que ha experimentado la sociedad hoy en día es la irrupción de identidades que tradicionalmente estuvieron sumergidas bajo los discursos dominantes de una identidad nacional excluyente y cerrada. De ahí la necesidad de crear otro medio y entorno educativo complementario: la llamada educación intercultural (López, 1997; González, 2004).

En México, el sistema educativo se ha ido adaptando a los requerimientos del mercado y a las necesidades de las grandes corporaciones privadas. En esa tendencia la universidad liquida (Bauman, 2004) casi se ha ido desvaneciendo. Desde la década de los setenta, y con más afán en los ochenta, se han privilegiado los métodos sobre el conocimiento universal. Incluso, se han

Rev. educ. super. sur glob-RESUR No3 En.-jul 2017 e006 ISSN 2393-6789 
creado universidades en donde el dominio de procesos industriales, tecnológicos y administrativos es lo más importante, tal es el caso de los institutos tecnológicos y algunas universidades politécnicas. Producir más con menos es la prioridad de hoy, producir para competir; al finalizar el ejercicio escolar cientos de escuelas superiores envían al mercado laboral a miles de egresados de distintas disciplinas que en realidad, de inmediato pasan a engrosar las filas del desempleo a lo que se suma un contexto sociopolítico global de una educación neoliberal (Flores-Crespo, 2006, 2006; Casillas y Santini, 2006).

La Constitución Política de los Estados Unidos Mexicanos menciona, en su artículo $3^{\circ}$, la equidad de la educación y en el artículo $2^{\circ}$, apartado A, fracción iv reconoce y garantiza el derecho de los pueblos y las comunidades indígenas a la libre determinación y en consecuencia, a la autonomía para preservar y enriquecer sus lenguas, conocimientos y todos los elementos que constituyen su cultura e identidad.

La interculturalidad es un concepto en construcción y polisémico, refiriéndose a una intención de ir más allá de la multiculturalidad o la pluriculturalidad. Essomba indica:

“...la interculturalidad es una práctica de vida diaria. Lo practican las personas concretas que buscan mayor igualdad y justicia social, no conformándose con lo que tienen. La responsabilidad de que la interculturalidad funcione es de la sociedad civil, son los habitantes de una comunidad los que tienen que respaldarla mediante sus actos. Las practicas interculturales deben ser un puente, no un muro, porque los seres humanos somos iguales en esencia y diferentes en existencia" (Essomba, 2006:11).

Lo anterior queda claro al analizar lo que ocurre con las culturas indígenas de México, partiendo de una historia de opresión y explotación que nos conduce a una realidad de mestizaje, misma que incluye serios intentos de asimilación tratando de integrar a los indígenas y su cultura dominante; la realidad intercultural supone una relación y una interacción entre grupos humanos con culturas diferentes, las cuales se dan en condiciones de igualdad entre ellas. La

Rev. educ. super. sur glob-RESUR N³ $\quad$ En.-jul 2017 e006 ISSN 2393-6789 
interculturalidad busca eliminar las diferencias al suponer una relación que incluya comprensión y respeto entre las culturas.

\section{La Universidad Intercultural de Chiapas}

El primero de enero de 1994 el Ejército Zapatista de Liberación Nacional (EZLN) se levantó en armas poniendo en el centro del debate nacional la situación de los pueblos indígenas. Es así, que la UNICh se convierte en un esfuerzo ideado desde el Estado mexicano con la finalidad de cumplir parcialmente las demandas de los indígenas chiapanecos. Por lo tanto, dicha universidad se ha caracterizado por incorporar el enfoque intercultural, que busca generar relaciones más armoniosas entre los diferentes. Busca reconocer la lengua y la cultura de los pueblos originarios y por lo tanto el corpus de conocimientos ambientales con los que cuentan. Fue así que el $1^{\circ}$ de diciembre del año 2004 se decretó la formación de la universidad intercultural de Chiapas, constituyendo la segunda universidad con el modelo de enseñanza superior intercultural en el país, situándose en la ciudad de San Cristóbal de las Casas de la región Altos de Chiapas.

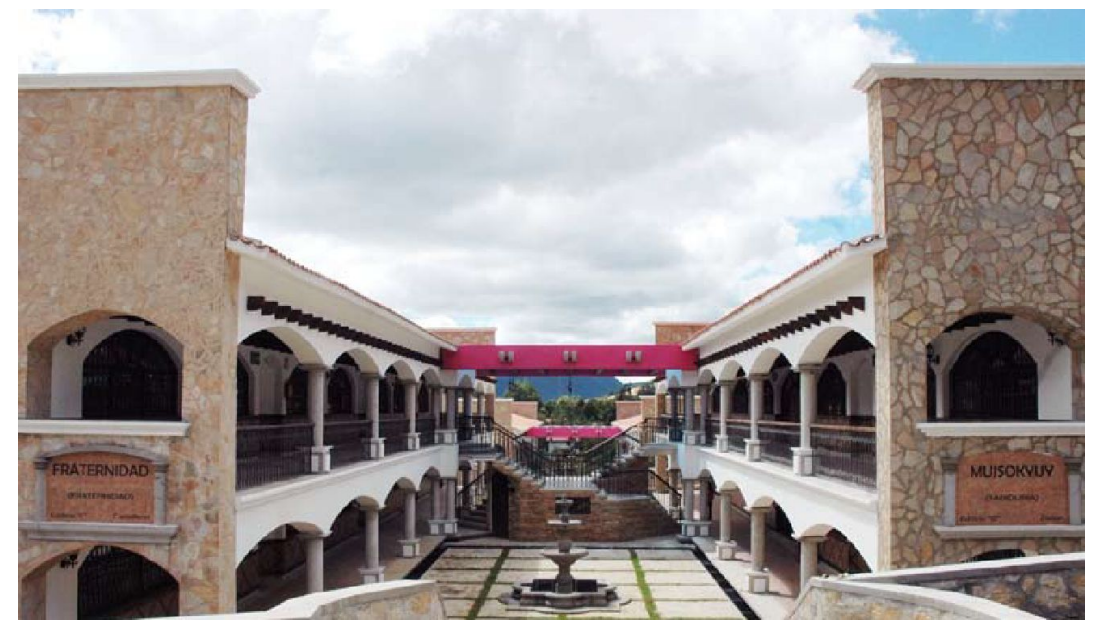

Figura 1. Instalaciones de la UNICh, San Cristóbal de Las Casas

La misión de la UNICh, es:

“...formar profesionistas con una sólida formación interdisciplinaria, conocimiento de las lenguas originarias y capacidad para el diálogo intercultural y de saberes, éticamente

Rev. educ. super. sur glob-RESUR N³ $\quad$ En.-jul 2017 e006 ISSN 2393-6789 
responsables de su compromiso social y representantes de la sociedad pluricultural mexicana, a través de programas educativos basados en el modelo educativo intercultural que promueve procesos de aprendizajes y construcción del conocimiento donde confluyan el saber científico y saberes tradicionales, donde el reconocimiento y revitalización de la lengua y la cultura se orienta a la construcción de una sociedad abierta a la diversidad encaminada a mejorar la calidad de vida, comprometida con el desarrollo social y económico sostenible local, regional y nacional" (Plan Institucional de Desarrollo 2008-2016:18).

En su corta vida, ha pasado por tres mapas curriculares: 2005, 2008 y 2011. En el año 2009, la UNICh obtuvo mayor cobertura en otros pueblos indígenas como en los municipios de Las Margaritas, Yajalón y Oxchuc y recientemente se ha concretado una sede más en Valle de Tulijá. La Unidad Académica Multidisciplinaria de Oxchuc, inició sus actividades académicas en el año 2009 en las instalaciones del Colegio de Ciencia y Tecnología (CECYT) No. 11 en el horario vespertino, ofertando las carreras de Desarrollo sustentable y Lengua y cultura. Desde sus inicios, se postuló como meta el establecimiento de un radio de incidencia educativa que abarcara la región tzeltal, es decir, una cobertura que brindara un aseguramiento de la pertinencia institucional, la diversificación de la oferta educativa y la atención de la demanda, no sólo para jóvenes del municipio de Oxchuc, sino de otros lugares como Chanal, Huixtán, Cancuc y Ocosingo. El personal inicial de la unidad académica, se conformaba por el coordinador académico, el coordinador administrativo, en responsable de servicios escolares, un encargado de sistemas informáticos y dos profesores de tiempo completo (uno para Desarrollo sustentable y el otro para lengua y cultura). Además de contar con una plantilla inicial de cinco docentes de asignatura que atendían a las dos licenciaturas.

La planta actual en términos de los docentes de asignatura se ha incrementado en un $50 \%$, que atienden la mayoría de ellos, en las dos licenciaturas. Los docentes que imparten clases en

Rev. educ. super. sur glob-RESUR No3 En.-jul 2017 e006 ISSN 2393-6789 
esta unidad, cuenta en su mayoría con estudios de maestría y algunos están en procesos de culminar el doctorado.

Desde el año 2005, la UNICh central de la ciudad de San Cristóbal de Las Casas ofertó cuatro licenciaturas pioneras: Desarrollo sustentable, Turismo Alternativo, Comunicación intercultural y Lengua y cultura registradas ante la SEP. Posteriormente, se aperturaron las carreras de Medicina con enfoque intercultural y Derecho intercultural en las diversas unidades académicas multidisciplinarias (cuadro 4).

Cuadro 4.

\begin{tabular}{ll}
\hline Unidades académicas & Programas educativos \\
\hline Unidad académica central San & Desarrollo sustentable, comunicación intercultural, Lengua \\
Cristóbal de Las Casas & y cultura, Turismo alternativo, Medicina intercultural y \\
& Derecho intercultural. \\
Unidad académica Las Margaritas & Lengua y cultura, Turismo alternativo y Derecho \\
Unidad académica Yajalón & intercultural. \\
Unidad académica Oxchuc & Lengua y cultura y Desarrollo sustentable \\
Unidad académica Valle del tulijá & Turismo alternativo y Desarrollo sustentable \\
\hline
\end{tabular}

Fuente: UNICH. Informe Institucional 2013

La carrera de Desarrollo sustentable, busca formar profesionales con alto sentido de responsabilidad con la sociedad y el medio ambiente y que contribuyen al desarrollo propio de sus familias y sus comunidades. Se espera consolidar una posición crítica ante los problemas de la sociedad en su conjunto y capaz de elaborar propuestas integrales de desarrollo en diferentes escalas de agregación a través de una sólida base teórica-metodológica, constituyéndose en un actor del desarrollo que promueve el diálogo intercultural entre la compleja diversidad de las

Rev. educ. super. sur glob-RESUR $N^{o} 3 \quad$ En.-jul 2017 e006 $\quad$ ISSN 2393-6789 
relaciones sociales, políticas y culturales, favoreciendo los procesos de vinculación comunitaria y el manejo sustentable de los recursos naturales, que le permita actuar como un conductor de procesos socioeconómicos donde media un profundo respeto hacia la naturaleza.

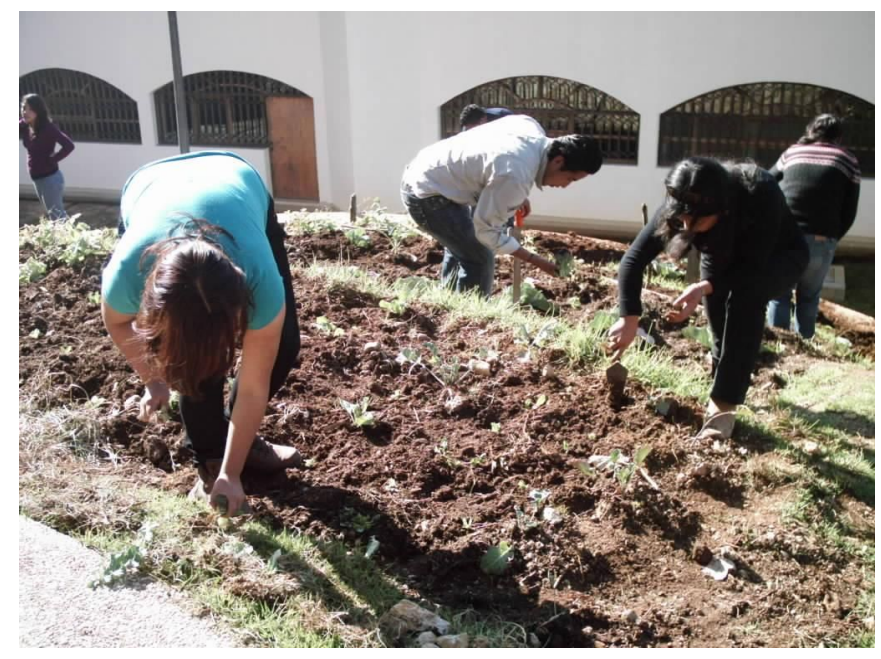

Figura 2. Estudiantes de la carrera de Desarrollo sustentable limpiando hortalizas

La carrera de Comunicación intercultural, implementa proyectos de comunicación que coadyuve a la revitalización de las lenguas originarias, las formas y expresiones artísticas y culturales para favorecer las relaciones entre las culturas; además, investiga problemáticas comunitarias para generar estrategias de comunicación que contribuya a la toma de decisiones y a la participación social y política. Los egresados, producirán material impreso en diferentes géneros periodísticos, audiovisual y multimedia para divulgar las identidades culturales y visibilizar la diversidad biocultural.

La carrera de Lengua y cultura, gestiona proyectos lingüísticos y culturales de desarrollo comunitario, basados en la investigación; además se desarrollan habilidades para fortalecer las lenguas de Chiapas a través de la generación de propuestas para su enseñanza, de la elaboración

Rev. educ. super. sur glob-RESUR N³ $\quad$ En.-jul 2017 e006 ISSN 2393-6789 
de recursos didácticos y de realizar traducciones. Se busca un promotor de la interculturalidad y la valoración de la diversidad cultural y lingüística.

La carrera de turismo alternativo, busca diseñar, gestionar y ejecutar proyectos turísticos en sus tres modalidades: ecoturismo, turismo rural y de aventura; además, promueve la organización de grupos de trabajo, la evaluación del potencial turístico de nuevos destinos y productos turísticos sustentables hacia la implementación de nuevos modelos de desarrollo turístico comunitario.

La carrera de Medicina intercultural, promueve formar recursos humanos con dominio de la atención médica general, en el manejo de ciencias y humanidades con una visión ética y profesional.

La carrera de Derecho intercultural, buscar formar recursos humanos capaces de mediar asuntos comunitarios por la vía conciliatoria aplicando los diversos sistemas normativos de la región, además de intervenir como asesor, representante, apoderado legal en asuntos jurídicos, administrativos y agrarios ante las instancias públicas, privadas, judiciales, legislativas y ejecutivas, haciendo valer en todo momento las reglas y prácticas autóctonas que pertenecen al derecho consuetudinario, el derecho positivo, tratados y convenios internacionales en el ámbito local, nacional e internacional.

Para el ingreso a la universidad, se da prioridad a aquellos estudiantes provenientes de pueblos indígenas. La matrícula de la universidad ha ido en crecimiento constante cada ciclo escolar (Gráfico 1), empezó con 662 estudiantes en el año 2005 hasta con 1824 estudiantes en el año 2014.

Gráfico 1. Matrícula de estudiantes

Rev. educ. super. sur glob-RESUR $N^{o} 3 \quad$ En.-jul 2017 e006 $\quad$ ISSN 2393-6789 


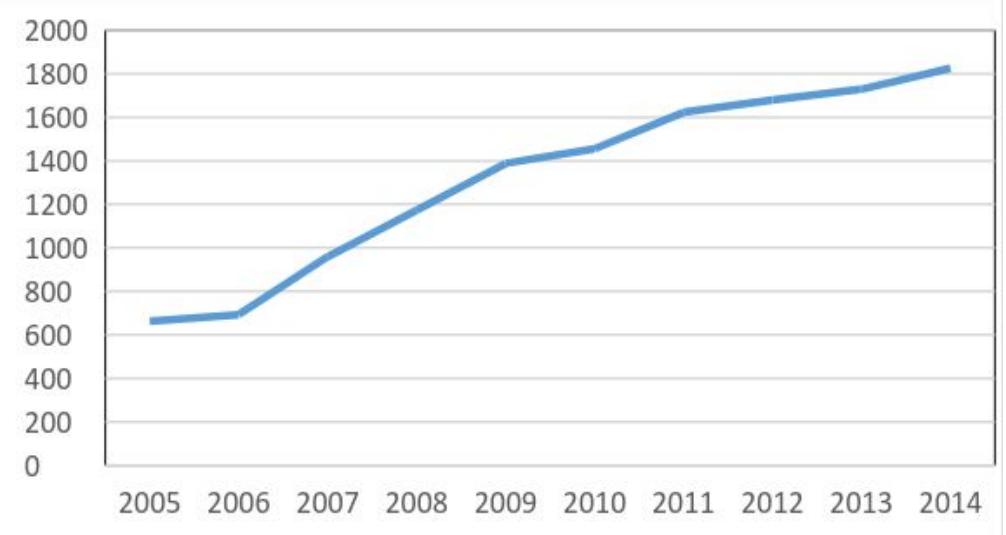

La mayor concentración de la matrícula de estudiantes, se encuentra en la unidad académica multidisciplinaria de San Cristóbal de Las Casas y la menor en la unidad académica Valle de Tulijá (Gráfico 2).

Gráfico 2. Distribución de la matrícula por unidad académica multidisciplinaria

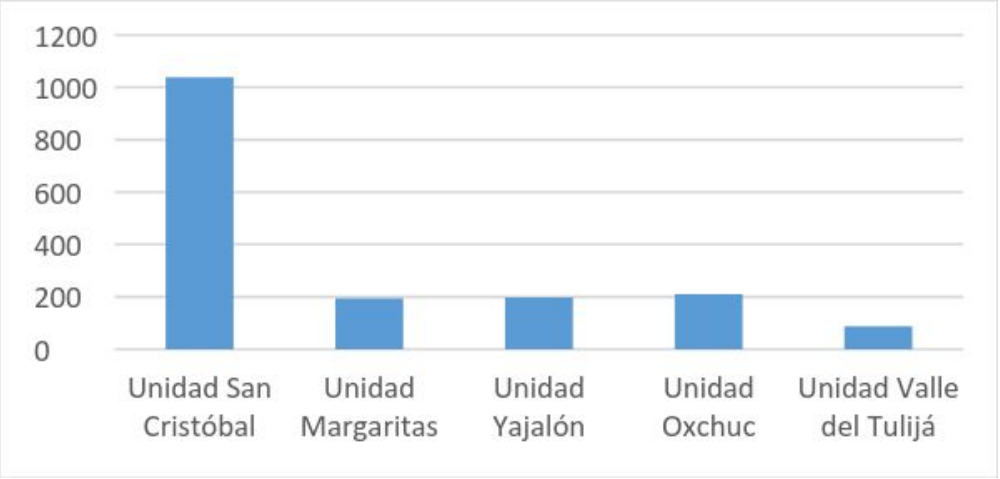

Rev. educ. super. sur glob-RESUR N³ $\quad$ En.-jul 2017 e006 ISSN 2393-6789 
La mayor cantidad de estudiantes están inscritos en la carrera de Lengua y cultura, seguido por Desarrollo sustentable y Turismo alternativo (Gráfico 3).

Gráfico 3. Matrícula por programa educativo

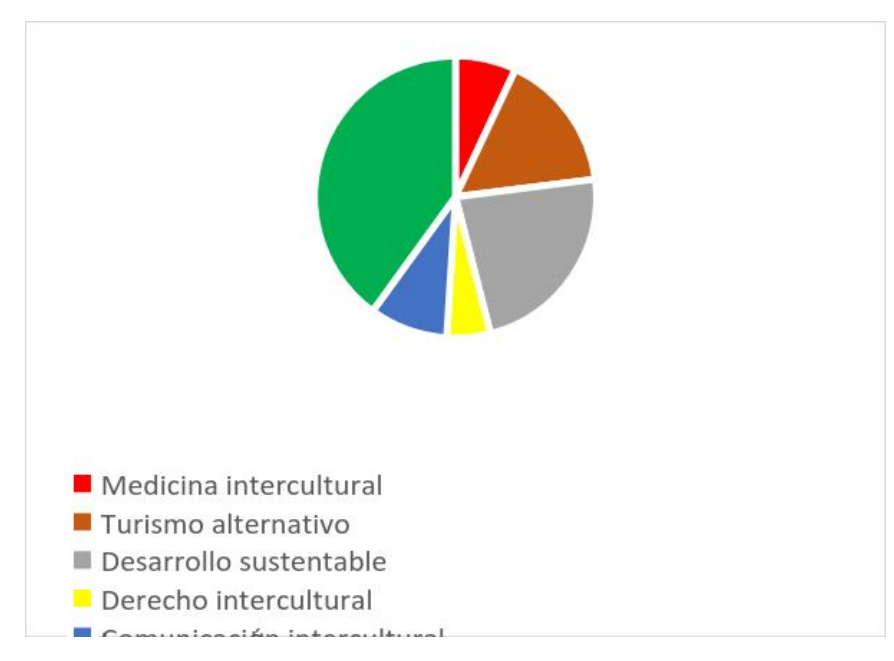

El equilibrio de género, se presenta de manera constante lo que significa que los roles en los pueblos originarios han ido cambiando, muestra de ello es la participación de la mujer en su rol como estudiante universitaria (Gráfico 4).

Gráfico 4. Distribución de la matrícula por sexo

Rev. educ. super. sur glob-RESUR $N^{o} 3 \quad$ En.-jul 2017 e006 $\quad$ ISSN 2393-6789 
La mayor parte de los estudiantes hablan una lengua originaria de los pueblos indígenas, del total de la matrícula solamente el $33 \%$ hablan la lengua castellana y el resto hablan alguna variante indígena (Gráfico 5).

Gráfico 5. Distribución de la matrícula por tipo de lengua
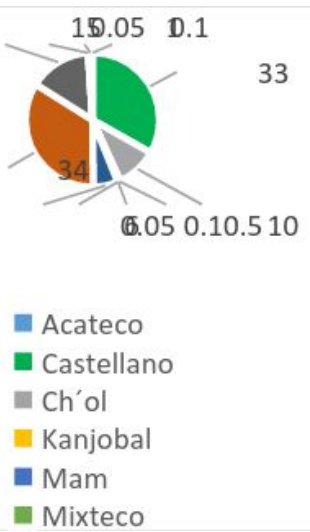

Hasta la fecha, existe un total de 32 plazas de Profesores de Tiempo Completo (PTC), 125 profesores de asignatura y 30 técnicos; de los PTC, cinco plazas están adscritas a las unidades académicas multidisciplinarias, con excepción de la unidad Valle del Tulijá. De los PTC, 11 tienen el grado de doctor representando el 34\% del personal académico, 21 tienen el grado de maestros en ciencias representando el 66\%, de los cuáles 12 estudian doctorado. En la universidad, existe una relación de 54/1 entre alumno y PTC (Plan Institucional de Desarrollo 2013-2024).

En el año 2012, 510 estudiantes realizaron actividades de vinculación en la modalidad de servicio social y 47 estudiantes en proyectos productivos. Los proyectos de vinculación, fueron financiados por la Comisión de Desarrollo Indígena en la convocatoria 2011. Se beneficiaron 41 municipios entre los que destacan: San Cristóbal de Las Casas, Las Margaritas, Yajalón, Oxchuc, Cancuc, Chenalhó, Cintalapa, Zinacantán, Chamula, Amatenango del Valle, Tenejapa, 
Larrainzar, Santiago el Pinar, Teopisca, Chalchihuitán, Huixtán, Ocosingo, Venustiano Carranza, Pantelhó, Sitalá, Aldama, Mitontic, Huitiupán, Mazapa de Madero, Motozintla, entre otros.

La Universidad Intercultural de Chiapas es un espacio de formación integral, que busca la interculturalidad y la sustentabilidad. En las licenciaturas en Desarrollo sustentable y Turismo alternativo, que dependen de la división de procesos naturales, se tiene incorporado un eje de formación (horizontal) a través del cual se busca generar aprendizajes ecológicos productivos por lo que los estudiantes conocen a profundidad la cuestión biológica y ecoproductiva. En el caso particular de la Licenciatura en Desarrollo sustentable cuenta con un eje de vinculación comunitaria y de integración de saberes, mediante en que se busca, generar nuevos saberes ambientales, fundamentalmente a partir del impulso de procesos de estancia, diagnóstico, reflexión, participación e interacción en una comunidad rural.

La creación de la Universidad Intercultural de Chiapas ha considerado la problemática del desarrollo y el análisis de lo económico, político, cultural y social del estado, en relación con el contexto nacional y mundial (estudio de factibilidad para la creación de la Universidad Intercultural de Chiapas, 2004), para establecer prioridades en la conformación de una oferta educativa pertinente a las necesidades locales.

En esta oferta educativa se trataba de atender la especificidad de las necesidades de desarrollo regional mediante una propuesta de formación específica. En el caso de Chiapas, las opciones de formación profesional se dirigieron a atender la problemática del desarrollo por medio de las licenciaturas en desarrollo sustentable y turismo alternativo, y en el ámbito cultural con las licenciaturas de lengua y cultura y en comunicación intercultural. Adicionalmente, la propuesta tomó en cuenta las nuevas orientaciones en educación superior tales como:

- Diversificación de la oferta educativa. Posibilidad de contar con diversas opciones de formación profesional acordes a las necesidades regionales.

- Pertinencia. Posibilidad de ofrecer respuestas adecuadas a las necesidades de formación profesional.

Rev. educ. super. sur glob-RESUR $N^{o} 3 \quad$ En.-jul 2017 e006 $\quad$ ISSN 2393-6789 
- Flexibilidad curricular. Incorporación de recursos académico-administrativos orientados a garantizar condiciones favorables de ingreso, permanencia, desarrollo académico y egreso de estudiantes.

- Aprendizaje centrado en el estudiante. Posibilidad de que el estudiante tome decisiones respecto a su propio proyecto de formación, que desarrolle autonomía en su proceso de aprendizaje y que aprenda por sí mismo de manera permanente (enfoque de aprender a aprender).

- Competencias profesionales. Expresan una relación entre los conocimientos, habilidades y actitudes que desarrollan los estudiantes y su desempeño satisfactorio de las actividades correspondientes a un ámbito profesional.

- Integralidad. Pretende que el estudiante desarrolle capacidad cognitiva y competencias para insertarse en contextos laborales diversos, que desarrolle autonomía en el aprendizaje, que fortalezca su identidad y dignidad, así como el fortalecimiento de valores que le permitan reconocerse como parte de su comunidad.

- Enfoque intercultural. Perspectiva que permita a los estudiantes reconocer la propia cultura, comprender y valorar la diversidad de otras prácticas culturales y favorecer la convivencia social.

- Calidad. Se caracteriza por el rigor académico, la pertinencia social y el logro de niveles comparables a los de otros de otras instituciones de educación superior.

- Vinculación teoría-práctica. Se expresa mediante la aplicación de conocimientos en el proceso de búsqueda de soluciones confrontando, valorando y utilizando los conocimientos tradicionales y modernos.

Asimismo, es necesario mencionar que la propuesta de este modelo educativo se desarrolla por medio de los siguientes ejes transversales: eje de lengua y cultura, expresa la cultura y valora la lengua; eje disciplinar, corresponde a las asignaturas y áreas de conocimiento específica del programa educativo; eje metodológico, corresponde a los procedimientos y procesos que

Rev. educ. super. sur glob-RESUR No3 En.-jul 2017 e006 ISSN 2393-6789 
corresponden al saber hacer y el eje axiológico, relacionado con los valores y actitudes que distinguen al modelo educativo.

Mediante estos cuatro ejes el proceso formativo va desarrollando, generando y revalorando diversos tipos de saberes que finalmente conforman el perfil profesional del estudiante que en términos de competencias representan tres tipos de saberes: saber conceptual, se refiere a los conceptos y palabras clave (conocimiento) y responde a la pregunta guía ¿cuáles son los conceptos o palabra clave vistos en clase?; saber hacer, dirigido al desarrollo de procesos y metodologías (habilidades) y saber ser, en torno a los valores (actitudes) ¿cómo se aplica en la forma de ser y vivir? Como los elementos que se han mencionado hasta el momento se observa la complejidad que implica la aplicación del modelo educativo así como su aplicación específica en un programa académico de desarrollo sustentable.

\section{EI desarrollo sustentable en la propuesta curricular de la UNICh}

Al igual que el concepto de desarrollo, el caso del desarrollo sustentable es un paradigma debido a que puede verse e interpretarse desde la economía, el desarrollo humano, las capacidades, el bienestar, etc. Es un sistema complejo ya que su planteamiento requiere considerar múltiples factores desde muy diversas dimensiones de análisis. Es por ello que el desarrollo sustentable no puede entenderse desde la visión de una sola disciplina, siendo la economía la que generalmente domina en el ámbito académico y político. Sin embargo, como bien sabemos, lo que se trata es que el desarrollo se estudie y analice desde una perspectiva integral que proponga a su vez mejores propuestas de desarrollo. El planteamiento curricular de la licenciatura en desarrollo sustentable de la UNICh propone una visión multidisciplinaria de la sustentabilidad mediante los tres ejes que lo caracterizan (ambiental-productivo, político-económico y social-cultural), para promover un proceso educativo en equilibrio donde se sensibilice y reflexione de manera integral sobre la relación entre población y ambiente. Dichos ejes se constituyen en esta propuesta curricular en las líneas de formación profesional que se desarrollan en forma horizontal a los

Rev. educ. super. sur glob-RESUR No3 En.-jul 2017 e006 ISSN 2393-6789 
ocho semestres y que caracterizan al desarrollo sustentable. En congruencia con la integridad que caracteriza a la propuesta educativa, en cada semestre los estudiantes tendrán la posibilidad de cursar asignaturas de las tres líneas de formación de manera simultánea.

Para el desarrollo del modelo educativo se requiere del uso y dominio de algunas estrategias para el aprendizaje significativo a partir de técnicas de integración grupal, análisis, organización, abstracción, comunicación, entre otras, indispensables para la integración y el desarrollo de una didáctica adecuada para el proceso de aplicación del mapa curricular a partir de tres ejes estratégicos:

Interculturalidad: técnicas para la revalorización de los conocimientos autóctonos y modernos, el reconocimiento de los sistemas de producción alternativa, la tecnología tradicional y moderna y la diversidad de las manifestaciones culturales. El reconocimiento a la diversidad de opiniones, el desarrollo de una actitud incluyente ante otros procesos productivos, organizacionales y culturales, entre otros.

Habilidades del pensamiento: técnicas para el desarrollo de habilidades del pensamiento superior, el análisis comparativo entre sistemas de producción, proyectos y formas de organización y producción, desarrollo de una actitud crítica ante situaciones reales, desarrollo de valores mediante la toma de conciencia social.

Comunicación: técnicas para fortalecer la capacidad para trabajar en equipo, el trabajo colaborativo, practicar y desarrollar actividades organizativas, la comunicación como base para la integración de grupos de trabajo e integración, el manejo de conflictos.

En todo diseño curricular se debe establecer una relación congruente tanto en forma longitudinal como vertical. El estudio de la sustentabilidad en esta propuesta contempla esta relación y en el caso del planteamiento vertical se cuenta con la línea de formación denominada "Integración de saberes y vinculación". Los cursos de integración de saberes que contempla la línea representan un espacio de discusión y análisis de los saberes adquiridos mediante temas-eje que varían en cada semestre, lo cual evita que los cursos sean un simple repaso de los temas

Rev. educ. super. sur glob-RESUR No3 En.-jul 2017 e006 ISSN 2393-6789 
vistos en otras asignaturas, ya que además contempla niveles crecientes de complejidad cognitiva que inician en el tercer semestre y hasta el octavo tomando como base la taxonomía de Bloom. Cabe mencionar que el modelo no se aplica a cada semestre en forma independiente, sino que el grado de complejidad se va incrementando conforme al avance semestral.

\section{Módulos agroecológicos para la vinculación comunitaria}

El programa titulado "Módulos universitarios de capacitación de tecnologías sustentables", como parte de las actividades de la asignatura de agroecología en el cuarto semestre del programa. Se propone con la finalidad de tener un espacio en el cual se puedan llevar a cabo algunas prácticas de producción agroecológica, que permitan el aprendizaje teórico-práctico de los alumnos(as) de la Licenciatura en Desarrollo Sustentable y Turismo Alternativo de la Universidad Intercultural de Chiapas. Además este programa funge como un espacio de generación y trasmisión de conocimientos para coadyuvar a reducir la problemática regional del deterioro ambiental y mejorar las condiciones de vida de las familias campesinas. En conjunto con los alumnos se busca diseñar agroecosistemas sustentables, por lo que se pretende formar equipos en las comunidades de procedencia de los estudiantes, quienes tendrán la responsabilidad de multiplicar, complementar y hacer fluir la información, mediante un proceso de diálogo con los productores(as), adecuando las propuestas productivas y definiendo conjuntamente planes de desarrollo local.

La UNICh pretende que sus alumnos(as) tengan las bases teóricas y prácticas para que en sus comunidades de origen, produzcan sus propios alimentos bajo un manejo agroecológico. Paralelo a ello, se busca también que el alumnado se forme, prácticamente, con los conocimientos para entender y enfrentar los problemas de las principales actividades agropecuarias de la región $\mathrm{y}$, de esa manera, se incorporen bien capacitados para trabajar en y con las comunidades campesinas.

Rev. educ. super. sur glob-RESUR $N^{o} 3 \quad$ En.-jul 2017 e006 $\quad$ ISSN 2393-6789 
El programa tiene como objetivo general el diseñar y construir un espacio socioambiental para el intercambio y asimilación de conocimientos entre estudiantes de la carrera de Desarrollo Sustentable y Turismo Alternativo, aprender las principales innovaciones agropecuarias y contar con un Centro de transferencia de conocimientos alternativos para los campesinos de la región. El programa contempla el desarrollo teórico-práctico de los siguientes módulos de producción: conservación de suelos, abonos orgánicos, camas biointensivas de producción y control de plagas. Se caracterizan por lo siguiente:

Módulo de conservación de suelos. Con el propósito de evitar la erosión hídrica y eólica en topografía inclinada y paralelamente conservar y aprovechar el suelo para la producción de hortalizas bajo un manejo agroecológico, se elaboraron terrazas en una área de aproximadamente $2,000 \mathrm{~m}^{2}$, con ayuda del aparato "A".

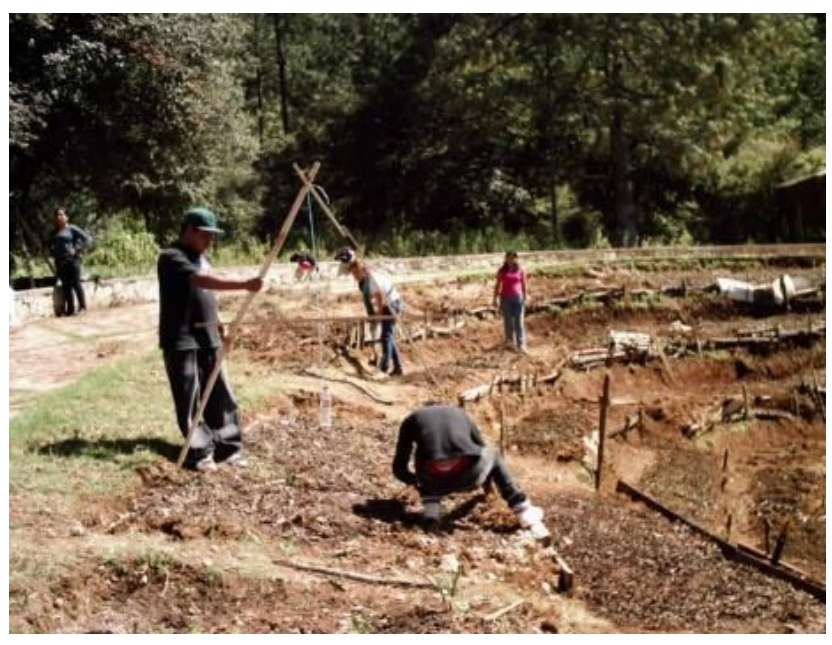

Rev. educ. super. sur glob-RESUR $N^{o} 3 \quad$ En.-jul 2017 e006 $\quad$ ISSN 2393-6789 
Figura 3. Estudiantes elaborando terrazas con el aparato "A"

Módulo de abonos orgánicos: los fertilizantes sintéticos como la urea, el sulfato de amonio y el triple 17 principalmente, deterioran la fertilidad del suelo (Kremlin, 1982:25). Los abonos orgánicos como el lombricultivo, el bocashi y la composta vienen a mejorar la fertilidad de los suelos agrícolas (Piamonte, 1992: 18).

El bocashi es un fertilizante orgánico que posee muchos nutrientes necesarios para los cultivos. Este fertilizante se obtiene mediante un proceso de descomposición aeróbica y hemofilica de residuos orgánicos a través de microorganismos que existen en los propios residuos. En el proceso de fabricación de este fertilizante orgánico, existen dos etapas: (1) la primera, es la estabilización donde la temperatura puede llegar alcanzar aproximadamente entre 70 y $75{ }^{\circ} \mathrm{C}$, debido al incremento de la actividad microbiana. Posteriormente, la temperatura del abono comienza a decrecer nuevamente, debido al agotamiento o disminución de la fuente energética que retroalimentaba el proceso. A partir de este momento, el abono pasa a la segunda etapa que es la maduración, donde la degradación de los materiales orgánicos se realiza por microorganismos con oxígeno todavía permanecen es más lenta, para luego llegar a su estado ideal para su inmediata utilización (Restrepo, 1998: 48).

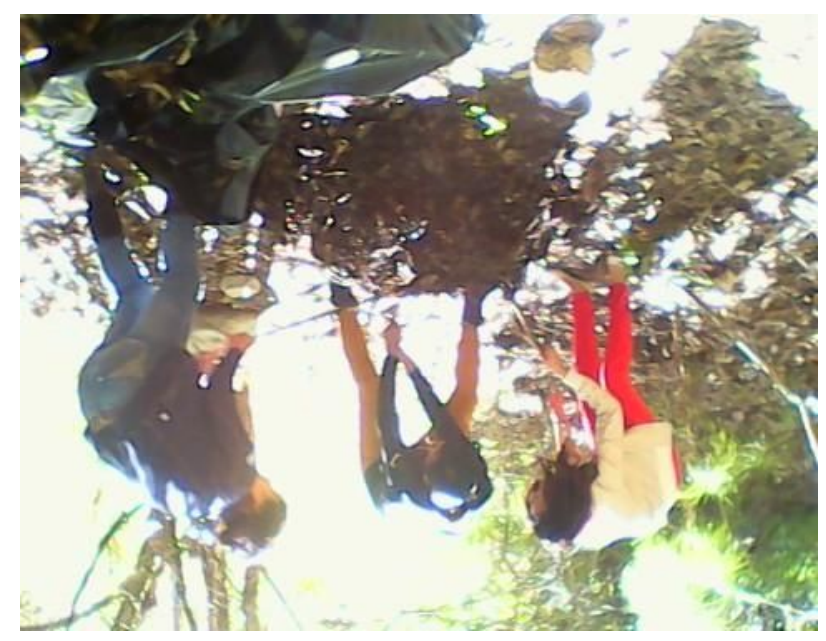

$\begin{array}{llll}\text { Rev. educ. super. sur glob-RESUR N } N^{\circ} 3 & \text { En.-jul } 2017 \text { e006 } & \text { ISSN 2393-6789 }\end{array}$ 
Figura 4. Estudiantes elaborando el abono orgánico Bocashi

La lombricultura o lombricompostaje es la cría masica, sistemática y controlada de lombrices. Es una técnica que involucra varios procesos biológicos, lo cual aceleran la transformación y mineralización de un residuo orgánico en descomposición y lo convierte en abono para las plantas. El lombricompostaje aprovecha las cualidades biológicas y fisiológicas de las lombrices para llevar a cabo la descomposición aeróbica de la materia orgánica. El producto del lombricompostaje, son las excretas, turrículos o heces fecales de las lombrices, este producto tiene la apariencia y olor de la tierra negra y fresca y es un sustrato estabilizado de gran uniformidad, contenido nutriental y con excelente estructura física, porosidad, aireación, drenaje y capacidad de retención de humedad. Durante el proceso de descomposición, no se producen desperdicios, malos olores y además, no requiere de equipos costosos y conocimiento experto (Capistrán et al., 2004: 80).

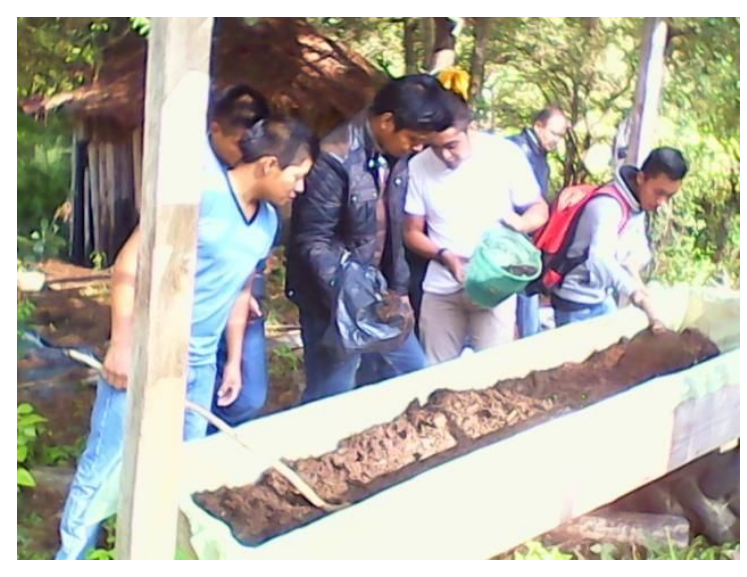

Figura 5. Estudiantes preparando el cajón para lombrices

Rev. educ. super. sur glob-RESUR No3 En.-jul 2017 e006 ISSN 2393-6789 
Módulo de camas biointensivas: una vez elaborada las terrazas, se preparó el suelo para sembrar hortalizas de la región. Bajo un manejo agroecológico, se sembraron especies criollas: jitomate rojo, tomate de cáscara, lechuga romana, chile jalapeño, cebolla, maíces criollos, zanahoria, rábano, cilantro, tomatillo silvestre, ruda, albahaca, hierbabuena, árboles de nim, chipilín, calabacita, frijol ejotero, canavalia, chayote, pepino, papas, entre otras.

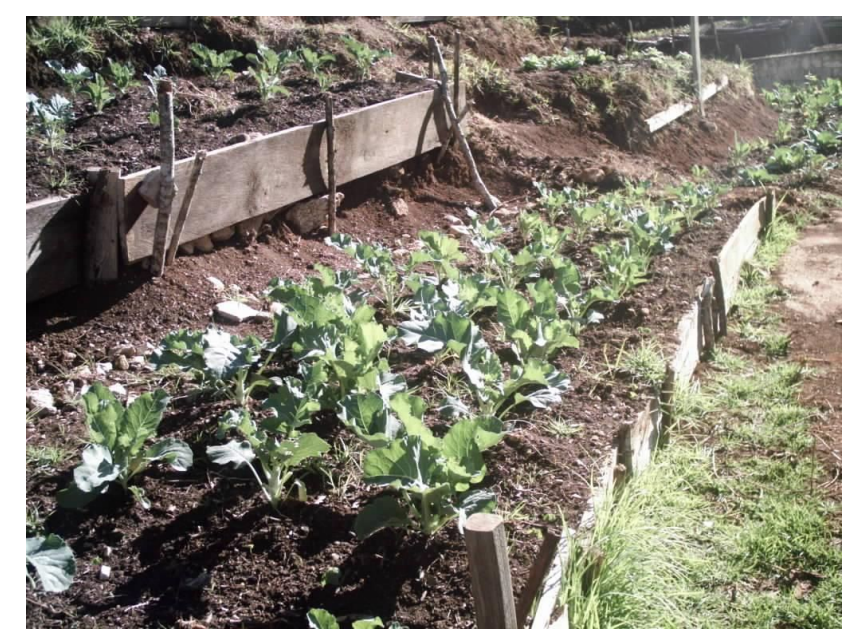

Figura 6. Camas biointensivas con cultivos de 45 días de siembra

Módulo de control de plagas: en cuestión de plagas y enfermedades, lo que se busca es prevenirlas antes de controlarlas. El diseño elaborado, justamente tiene el objetivo de disminuir los problemas de plagas y enfermedades que nos pudieran dañar a nuestros cultivos. Sin embargo, para tener una mejor producción se estará monitoreando los agroecosistemas para detectar los posibles insectos plagas y los patógenos portadores de enfermedades. Se tiene pensado para el caso de presencia de plagas, elaborar un programa de Manejo Integrado de Plagas (MIP). Por MIP estamos entendiendo al control racional de insectos plagas, basados en la biología y ecología trabajando junto

Rev. educ. super. sur glob-RESUR No3 En--jul 2017 e006 ISSN 2393-6789 
con la naturaleza en vez de en contra de ella. Con el MIP, prevenimos y controlamos las plagas considerando a todas las posibles estrategias amigables con la naturaleza, de tal forma que no dañe a los componentes de un agroecosistema.

Figura 7. Estudiantes colocando la trampa amarilla para el control de mosquita blanca

Dichos módulos de producción se han establecido al interior de las instalaciones de la Universidad Intercultural de Chiapas y como parte de las actividades de la asignatura de Agroecología, se tienen que replicar en las comunidades periféricas de la región.

\section{Proceso de vinculación comunitaria-desarrollo local}

Desarrollar los módulos agroecológicos en las comunidades rurales indígenas no ha sido nada fácil, ya que los campesinos están mal acostumbrados a usar los agroquímicos en sus cultivos y además significan actividades más fáciles de aplicación.

Actualmente, se ha trabajado con algunas organizaciones productivas de la región, como es el caso de la organización "Ivestic", ubicada en el municipio de San Cristóbal de Las Casas, que

\begin{tabular}{llll}
\hline Rev. educ. super. sur glob-RESUR N $N^{\circ} 3$ & En.-jul 2017 e006 & ISSN 2393-6789
\end{tabular} 
está conformada por 12 campesinos indígenas que se dedican al cultivo de flores, de rosas y frutales bajo un manejo en transición agroecológica.

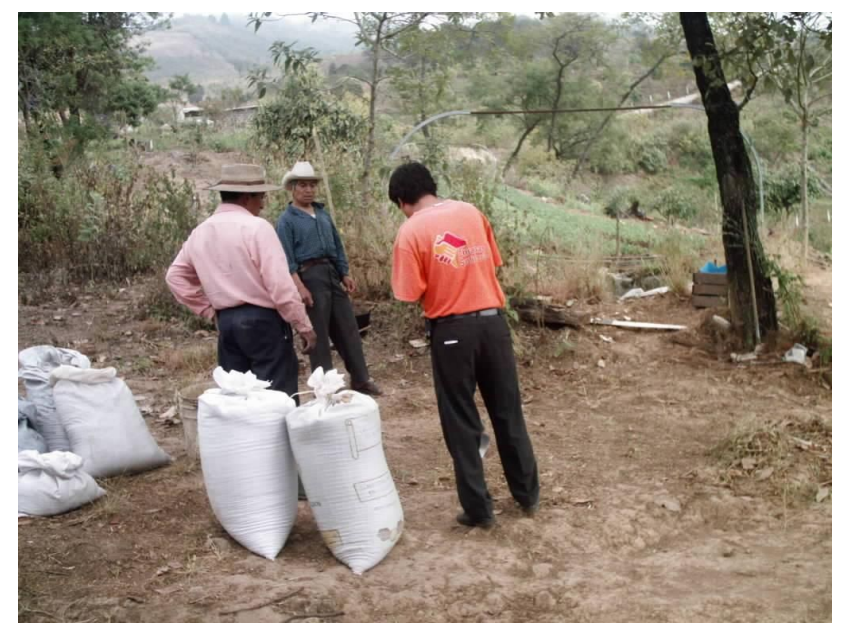

Figura 8. Integrantes de la organización elaborando el abono orgánico Bocashi

Otra experiencia de vinculación e intercambio de conocimientos tradicionales y científicos, se ubica en el municipio de Teopisca, en una huerta de producción de aguacate "Hass", bajo principios agroecológicos con tendencia hacia una agricultura orgánica.

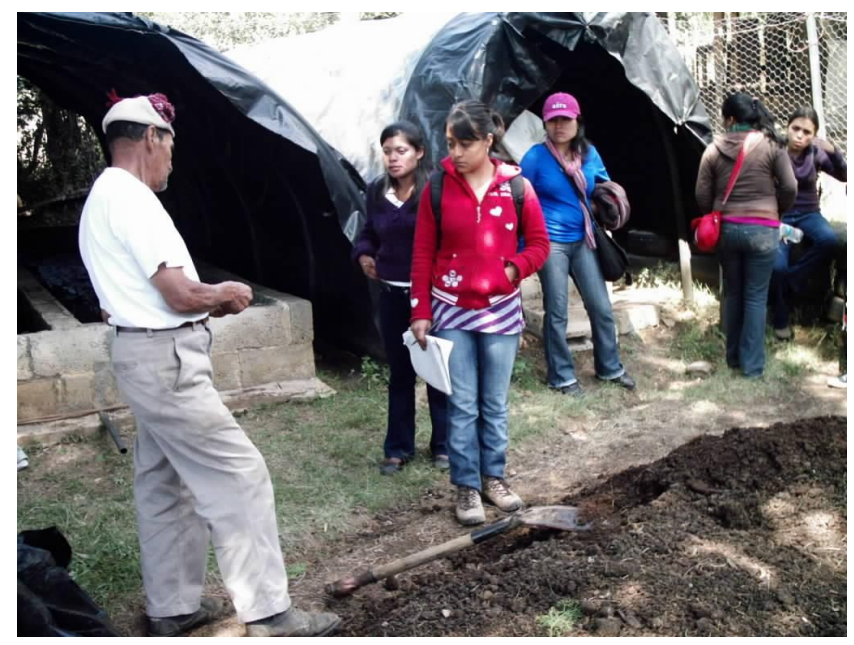

Figura 9. Visita de estudiantes a la huerta de aguacate de don Melecio

Rev. educ. super. sur glob-RESUR N³ $\quad$ En.-jul 2017 e006 ISSN 2393-6789 


\section{Alcances y Retos}

La propuesta curricular para en la licenciatura en desarrollo sustentable sigue construyéndose. Partimos de una propuesta docente integral tomando los ejes del desarrollo sustentable como plataforma de las líneas de formación académica. Buscamos la formación integral del estudiante mediante cursos simultáneos de los tres ejes durante el desarrollo de cada semestre, pero sobre todo buscamos la integración de saberes por medio del diálogo intercultural dentro y fuera del aula donde se contrastan todo tipo de saberes. Además, las prácticas de campo son un espacio primordial para fortalecer dicho diálogo de saberes.

Todo lo anterior implica el seguimiento pedagógico y la elaboración de indicadores que permitan valorar el avance del modelo educativo que en el fondo del asunto se resume en la promoción y el derecho a una vida digna a partir de la comunidad y en profunda convivencia con la naturaleza.

Según los objetivos propuestos en este trabajo, hay algunos alcances importantes que se desarrollaron en la parte anteriormente descrita. Son muchos los retos que faltan por concretar, hay dificultades para lograr comparar cabalmente lo logros entre los proyectos del diseño curricular y los aspectos que se observaron entre el plano de proyecto y algunos resultados esperados en el año de 2016. Sin embargo se pudo observar que entre los aspectos observados en el plano del diseño y en el nivel de resultados. Lo que explica que en el primer plano se incluyen una serie de razones que condujeron a la construcción de las aspiraciones. Mientras tanto en el resultado se toma en cuenta la participación de un conjunto de elementos que se corresponden con las clases de más fuerte poder, que fueron necesariamente aquellos grupos de docentes y directivos que se fusionan con el gobierno federal en turno.

\section{A manera de conclusión}

Rev. educ. super. sur glob-RESUR N³ $\quad$ En.-jul 2017 e006 ISSN 2393-6789 
La propuesta curricular para la licenciatura en desarrollo sustentable sigue construyéndose. Partimos de una propuesta docente integral tomando los ejes del desarrollo sustentable como plataforma de las líneas de formación académica.

Buscamos la formación integral del estudiante mediante cursos simultáneos de los tres ejes durante el desarrollo de cada semestre, pero sobre todo se promueve la integración de saberes en el aula y fuera de ella donde se contrastan conocimientos de los productores comunitarios y las teorías que aprenden los estudiantes en sus convivencias con los profesores. Además, las prácticas de campo son un espacio primordial para fortalecer dicho diálogo de saberes.

Bajo este panorama, el principal reto que enfrenta nuestro modelo educativo para el desarrollo sustentable es el cumplimiento del perfil de egreso del estudiante, el cual establece que ellos deben: conocer las bases teóricas para el manejo de los recursos naturales; conocer los problemas y necesidades de la población; saber los principios de la interculturalidad y de las formas de interrelación y vinculación comunitaria; promover los principios de la sustentabilidad; elaborar, gestionar, promover y evaluar proyectos de desarrollo sustentable; detonar procesos participativos entre los diversos actores de la sociedad; apreciar la vida comunitaria e interés por mejorar las condiciones de vida de la población; promover el diálogo intercultural para su pertinencia e identidad cultural.

Todo lo anterior implica el seguimiento pedagógico y la elaboración de indicadores que permitan valorar el avance del modelo educativo que en el fondo del asunto, se resume en la promoción y el derecho a una vida digna a partir de la comunidad y en profunda convivencia con la naturaleza.

Como elemento metodológico debe destacarse que, se comprende que en una década (2005-2015) de operación de la UNICh, hay más retos que metas cumplidas. Como suele suceder en la valoración de los proyectos sociales o socioeducativos, el tiempo de operación es muy corto para fundamentar juicios de calificación. Valorar un proyecto educativo con una década de vida

Rev. educ. super. sur glob-RESUR N³ $\quad$ En.-jul 2017 e006 ISSN 2393-6789 
es hasta, en cierta forma, un riesgo, porque efectivamente es difícil apreciar sus resultados porque muchas de las acciones se ubican en el largo plazo, es decir en un lapso de entre 20 o 25 años. Sin embargo en este primer ejercicio, se obtiene una idea o una tendencia acerca del futuro de las universidades interculturales en México, particularmente, la UNICh.

\section{Referencias bibliográficas}

Altieri, Miguel Ángel (1987). Agroecology: the scientific basis of alternative agriculture. Boulder colorado: Westview Press.

Barabas, Alicia M. (2006). "Notas sobre multiculturalismo e interculturalidad", en Diversidad y reconocimiento. Aproximaciones al multiculturalismo y la interculturalidad. Suplemento del boletín diario de campo. México.

Bauman Sygmunt (2004). La universidad líquida, Trokel, Paris.

Bruckman, Mónica y Dos Santos, Theotonio (2008). Los movimientos sociales en América Latina: Un balance histórico (en línea). Disponible en: http://www.cetri.be/spip

Casillas Muñoz, María de Lourdes y Santini Viilar, Laura (2006). Universidad intercultural. Modelo educativo. Ciudad de México: CGEIB-Secretaría de Educación Pública.

Essomba, Miguel Ángel (2006). Construir la escuela intercultural, Col. Biblioteca de Aula, Barcelona: Grao.

Flores-Cresco, Pedro (2006). Los retos de México en el futuro de la educación. Ciudad de México: Consejo de Especialista para la Educación.

González, O. Felipe (2004). "La construcción de un modelo de educación superior intercultural. La Universidad Intercultural”. Ciencia Ergo Sum. Universidad Autónoma del Estado de México, Vol. 11, Núm. 3, pp.303-307.

INEGI (2010). Censo de población y vivienda 2010. México: Instituto Nacional de Estadística y Geografía.

Kremlin, R. (1982). Plaguicidas modernos y su acción bioquímica. México: Limusa.

Rev. educ. super. sur glob-RESUR N³ $\quad$ En.-jul 2017 e006 ISSN 2393-6789 
López, L. E. (2009). "Interculturalidad, educación y política en América Latina: perspectivas desde el sur. Pistas para una investigación comprometida y dialogal”, en L. E. López (editor), Interculturalidad, educación y ciudadanía. Perspectivaslatinoamericanas. La Paz Bolivia: Plural Editores/Funproeib-Andes.

Martínez G.G, Victorino R.L. Reyes R.A. Coordinadores (2012). Diálogos entre saberes. Innovación educativa, educación ambiental e Interculturalidad. Porrúa, México.

Piamonte, R. (1992). Contribuicao ao desenvolvimento de novos métodos para asubacao verde. Botucatu: Instituto Biodinamico.

Piamonte, René (1992). Contribuicao ao desenvolvimento de novos métodos para asubaçao verde. Botucatu: Instituto Biodinamico.

Sevilla, Guzman and Woodgates (1997). "Sustainable rural development: forma industrial agriculture to agroecology", in M. Redclift and G. Woodgate (Coord.), Theinternational handbook of environmental sociology. Cheltenham.

UNICH. Informe Institucional 2013, UNICH, Chiapas, México.

UNICH (2008) Plan Institucional de Desarrollo 2008-2016, Chiapas, México.

Victorino R.L. y Atriano, M.R.A. (2015). "Las maestras rurales y su papel en los procesos de lucha para alcanzar la igualdad entre los géneros" en Velázquez R.E.B, Quintero S.M.L. Coordinadoras (2015). El reencuentro con la naturaleza: voces femeninas en el tiempo, UAEM-Castellanos Editores, México, pp.177-198.

Victorino, R.L. y Atriano, M. R.A. (2014). "El enfoque intercultural en la educación superior para prevenir y disminuir la exclusión social de los indígenas" en Velázquez R.E.B. Quintero S.M.L. Coordinadoras (2014) La transformación de la sociedad opresora, Porrúa-UAEM, México, pp. 101-122.

Victorino, R.L. (2016). Corrientes socioeducativas y teoría del sujeto social universitario, SOMEC, Castellanos editores-UACh, México.

Rev. educ. super. sur glob-RESUR $N^{o} 3 \quad$ En.-jul 2017 e006 $\quad$ ISSN 2393-6789 
Rev. educ. super. sur glob-RESUR $N^{\circ} 3 \quad$ En.-jul 2017 e006 ISSN 2393-6789 